\title{
Characterization of mAb6-9-1 monoclonal antibody against hemagglutinin of avian influenza virus H5N1 and its engineered derivative, single-chain variable fragment antibody
}

\author{
Róża Sawicka1', Paweł Siedlecki', Barbara Kalenik1, Jan P. Radomski², Violetta Sączyńska³, \\ Anna Porębska³, Bogusław Szewczyk ${ }^{4}$, Agnieszka Sirko' ${ }^{1}$ and Anna Góra-Sochacka ${ }^{1 凶}$
}

1 Institute of Biochemistry and Biophysics, Polish Academy of Sciences, Warsaw, Poland; 2 Interdisciplinary Centre for Mathematical and Computational Modelling, Warsaw University, Warsaw, Poland; ${ }^{3}$ Institute of Biotechnology and Antibiotics, Warsaw, Poland; ${ }^{4}$ Department of Recombinant Vaccines, Intercollegiate Faculty of Biotechnology, University of Gdansk and Medical University of Gdansk, Gdańsk, Poland

\begin{abstract}
Hemagglutinin (HA), as a major surface antigen of influenza virus, is widely used as a target for production of neutralizing antibodies. Monoclonal antibody, mAb6-9-1, directed against HA of highly pathogenic avian influenza virus A/swan/Poland/305-135V08/2006(H5N1) was purified from mouse hybridoma cells culture and characterized. The antigenic specificity of mAb6-9-1 was verified by testing its cross-reactivity with several variants of HA. The mimotopes recognized by $\mathrm{mAb} 6-9-1$ were selected from two types of phage display peptide libraries. The comparative structural model of the HA variant used for antibody generation was developed to further facilitate epitope mapping. Based on the sequences of the affinity- selected polypeptides and the structural model of HA the epitope was located to the region near the receptor binding site (RBS). Such localization of the epitope recognized by mAb6-9-1 is in concordance with its moderate hemagglutination inhibiting activity and its antigenic specificity. Additionally, total RNA isolated from the hybridoma cell line secreting mAb6-9-1 was used for obtaining two variants of cDNA encoding recombinant single-chain variable fragment (scFv) antibody. To ensure high production level and solubility in bacterial expression system, the scFv fragments were produced as chimeric proteins in fusion with thioredoxin or displayed on a phage surface after cloning into the phagemid vector. Specificity and affinity of the recombinant soluble and phage-bound ScFv were assayed by suitable variants of ELISA test. The observed differences in specificity were discussed.
\end{abstract}

Key words: influenza virus, monoclonal antibody, scFv, hemagglutinin, antigenic epitope

Received: 23 March, 2016; revised: 07 October, 2016; accepted: 14 October, 2016; available on-line: 07 December, 2016

\footnotetext{
e-mail: annag@ibb.waw.pl

Abbreviations: $\mathrm{CDR}$, complementarity determining region; $\mathrm{HA}$, hemagglutinin; $\mathrm{HI}$, hemagglutination inhibition test; HRP, Horseradish Peroxidase; $\mathrm{mAb}$, monoclonal antibody; PBS, phosphate-buffered saline; pfu, plaque forming unit; scFv, single-chain variable fragment antibody; RBS, receptor binding site; $\mathrm{VH}$, variable region of the heavy chain; $V L$, variable region of the light chain
}

\section{INTRODUCTION}

Antibodies are used in many research applications, as well as in various diagnostic assays and medical treatments. Hybridoma technology enables production of huge amounts of monoclonal antibodies (mAb) against the target antigen but it is a laborious, time consuming and expensive technique. Growing demand for recombinant antibodies and their derivatives prompted the development of various expression platforms. Up to date many attempts of their production in mammalian cells (Ho et al., 2006), insect cells (Choo et al., 2002), plants (Dobhal et al., 2013), yeast or bacteria (Rouet et al., 2012) have been reported. The derivatives of antibodies such as the antigen-binding fragment (Fab) or the single chain variable fragment $(\mathrm{scFv})$ are of particular interest. Their reduced structures and molecular weights facilitate expression and purification process. The $\mathrm{scFv}$ fragment consists of variable regions of heavy $(\mathrm{VH})$ and light (VL) chains of immunoglobulin connected by a flexible linker (Kalenik et al., 2014). The scFv can be modified by conjugation to drugs or toxins and may penetrate the blood and tissue more rapidly than a complete antibody (Cao et al., 2012a; Oriuchi et al., 2005). Easiness of modification gives many possibilities of direct or indirect detection of this protein and facilitates manipulations of its activity (affinity to antigens). These properties are especially important for such targets as rapidly evolving surface antigens of influenza virus.

The influenza viruses belong to Orthomyxoviridae family. Their genome consists of a single- stranded negative sense RNA. Due to the antigenic drift and shift, resulting frequently in novel mutants and re-assortants that are able to cross the species barriers, the influenza viruses are still a serious global problem for humans and their livestock, especially for poultry and pigs. Highly pathogenic avian $\mathrm{H} 5 \mathrm{~N} 1$ influenza viruses are responsible for serious economic losses to the poultry industry and have caused numerous threats to public health. Since 1996 when H5N1 virus was isolated for the first time in China it has spread rapidly and continued to evolve, which resulted in a periodic emergence of new phylogenetic groups in several regions of the world. Currently, $\mathrm{H} 5 \mathrm{~N} 1$ viruses are divided into multiple clades and subclades based on antigenic variation (Smith et al., 2012; Smith et al., 2015; Li et al., 2013). Hemagglutinin (HA), a strong surface antigen, is synthesized as a precursor, HA0 molecule, which is cleaved into two subunits, HA1 and HA2 (Szewczyk et al., 2014). The HA1 subunit encompasses the membrane-distal globular head containing the receptor binding site (RBS) and the vestigial esterase domain. The HA2 subunit encompasses the stalk region (Velkov, 2013). The main functions of HA during virus infection are attachment of the virus to the host cell re- 
ceptor and mediation of the fusion of the virus envelope with the endosomal membrane after endocytosis. This glycoprotein is a major target for anti-virus antibodies and a subject of high antigenic variability (antigenic drift). Identification and characterization of its antigenic epitopes is needed for understanding of the molecular mechanisms of antigenic drift and viral immune escape, which in turn might result in better selection of the vaccine strains. The reactivity of selected monoclonal antibodies with escape mutants of $\mathrm{H} 5 \mathrm{HA}$ were intensively studied but the map of $\mathrm{H} 5$ antigenic epitopes is still incomplete. Most of the characterized mAbs against $\mathrm{HA}$ from $\mathrm{H} 5 \mathrm{~N} 1$ virus recognize the epitopes localized in the globular head of HA, most of them overlap the RBS and are isolate-specific, (e.g. Cao et al., 2012b; Du et al., 2013; Wu et al., 2014), while antibodies binding to the stem region have often a broad activity (Tan et al., 2015; Zuo et al., 2015). Extensive review of H5 HA antigenic sites was provided by (Velkov et al., 2013) who described the anti-HA antibodies divided into 3 groups: (i) RBS selective, (ii) non-RBS, membrane-distal globular domain selective and (iii) HA2 selective.

The mAb6-9-1 is a monoclonal mouse antibody secreted by the hybridoma cell line 6-9-1, which was generated by us from immune cells of a mouse immunized with the recombinant $\mathrm{H} 5 \mathrm{HA}$ from A/swan/ Poland/305-135V08/2006 (H5N1). We described usage of Fab (antigen-binding fragment) fragment of mAb69-1 in electrochemical immunosensor detecting HA from H5N1 viruses (Jarocka et al., 2014). It was applied for detection of HA (17-530 aa) from A/Bar-headed Goose/Qinghai/12/05(H5N1) and HA1 subunit of HA from two strains of H5N1 viruses: A/swan/Poland/305135V08/2006 and A/Vietnam/1194/2004 Jarocka et al., 2014). The strongest binding was observed for the long HA from A/Bar-headed Goose/Qinghai/12/05(H5N1) with the detection limit of $2.2 \mathrm{pg} / \mathrm{mL}$. The successful application of the derivatives of this antibody prompted us to perform its broaden characterization, especially mapping of an epitope recognized by mAb6-9-1 on the structural model of the HA from A/swan/Poland/305135V08/2006 (H5N1). Moreover, we described cloning, expression and characterization of recombinant $\mathrm{scFv}$ fragments derived from the mAb6-9-1. The specificity of two variants (VLVH and VHVL) of $\mathrm{scFv}$ produced in fusion with thioredoxin were evaluated by indirect and sandwich ELISA assays and confirmed with the $\mathrm{scFv}$ variant produced by the phage display method.

\section{MATERIALS AND METHODS}

Hybridoma propagation and monoclonal antibody purification. Mouse hybridoma cell line 6-9-1 that secreted monoclonal antibody against hemagglutinin from A/swan/Poland/305-135V08/2006 (H5N1) was generated from splenocytes obtained after immunization of mice with the recombinant H5 HA. The hybridoma cells were maintained in RPMI-1640 medium (20 mM L-glutamine, $10 \%$ fetal bovine serum, $\left.5 \% \mathrm{CO}_{2}, 37^{\circ} \mathrm{C}\right)$. The mAb6-9-1 was purified from culture supernatant using protein $G$ (ThermoScientific).

Comparative modeling of HA from A/swan/ Poland/305-135V08/2006 (H5N1). Structures suitable for modeling were chosen from a default BLAST search against the PDB database (accessed February 5th, 2015), with respect to the serotype (H5), X-ray resolution, and sequence length. Two structures emerged through these criteria: 2ibx (A/Vietnam/1194/2004 (H5N1)) and 2fk0
(A/Vietnam/1203/2004 (H5N1)), both having over $90 \%$ sequence identity. Preparation of X-ray structures was done in Chimera (v1.11) and consisted of deletion of non-standard residues and $\mathrm{HOH}$ molecules, $\mathrm{N}$ - and C-terminal fix and incomplete side chains repair. Both structures were aligned with MatchMaker (Meng et al., 2006) and the most similar chains were used as templates; 2IBX_A, 2IBX_B, 2FK0_K, 2FK0_L. Modeler software (Sali \& Blundell, 1993) was used throughout the modeling process. Ten different models were generated and manually evaluated with respect to hydrogen bond patterns, AA contact and clashes and Ramachandran plot. The best model was subjected to a minimization procedure using AMBER force field (ff14SB) and a steepest descent procedure to relieve steric hindrances

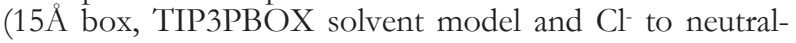
ize the structure).

Epitopes mapping. Epitope recognition by purified mAb6-9-1 was determined using two Phage Display Peptide Libraries, Ph.D. ${ }^{\text {TM }}$-C7C and Ph.D. ${ }^{\text {TM }}$-12 (New England Biolabs) according to the manufacturer's protocol (Ph.D Phage Display Libraries, version 1.2, New England Biolabs). Briefly, the 96-well plates (Maxisorb Nunc) were coated with purified mAb6-9-1 (7.5 $\mu \mathrm{g}$ / well) and incubated at $4^{\circ} \mathrm{C}$ overnight. After washing with TBST (TBS $+0.1 \%$ Tween-20) and blocking with BSA solution $(5 \mu \mathrm{g} / \mathrm{ml})$, the titrated phage peptide library (Ph.D. TM-C7C or Ph.D. ${ }^{\text {TM-12) }}$ was added to each well $\left(10^{7}-10^{8}\right.$ plaque forming units (pfu) in $\left.100 \mu \mathrm{l}\right)$ and incubated for 60 minutes at room temperature. Unbound phages were discarded, plates washed 10 times with TBST and bound phages were eluted with $0.2 \mathrm{M}$ glycine$\mathrm{HCl}$, (pH 2.2), $1 \mathrm{mg} / \mathrm{ml}$ BSA. In a parallel experiment the OET protein (see below, Antigens and antibodies used in ELISA) at concentration $0.8 \mathrm{mg} / \mathrm{ml}$ was used for phage elution. Eluted phages were amplified and taken to the next panning rounds. After three rounds of panning several phage clones were randomly selected, subjected to phage ELISA, (performed according to the protocol described in Ph.D Phage Display Libraries, New England Biolabs) to confirm their binding capacity with mAb6-9-1. DNA isolated from individual phage clones was sequenced and amino acid sequence of the corresponding peptides determined. The Pepitope server (http://pepitope.tau.ac.il/) (Mayrose et al., 2007a) was used to map the selected peptides onto the $3 \mathrm{D}$ model of HA from A/swan/Poland/305-135V08/2006 (H5N1). Using the consensus methodology, both PepSurf (Mayrose et al., 2007b) and Mapitope algorithms (Bublil et al., 2007) were executed and their results combined into a single prediction including only residues predicted to be a part of the epitope in both algorithms. Mapping results were compared with the surface of the H5 HA structural model using UCSF Chimera (v1.11).

Synthesis of the scFv cDNA. Primers. Sequences of primers used in the experiment of $\mathrm{scFv}_{\mathrm{v}}$ synthesis and cloning are listed in the Supplementary file Table S1 at www.actabp.pl.

Synthesis of VH and VL regions. Sequences encoding the variable regions of light (VL) and heavy (VH) chains were obtained according to the method and procedure described by (Ladiges \& Osman, 2001). The main steps of procedure are described compendiously below. Total RNA was isolated from the hybridoma cell line 6-9-1 using RNeasy Plus Micro Kit according to the manufacturer's instructions (Qiagen). $6.5 \mu \mathrm{g}$ of isolated RNA and oligo (dT)15 primer were used to synthesize blunt-ended cDNA using the cDNA Synthesis System (Roche). Next, the PCR OG/GO linker was ligated to 
the cDNA by T4 DNA ligase (Invitrogen). The resulting cDNA was used to amplify $\mathrm{VH}$ and VL fragments using Easy-A High-Fidelity PCR Master Mix (Agilent Technologies) supplemented with the sets of primers, $\mathrm{OG}$ and IgGkapp or OG and IgG1HC1 for VL and VH synthesis, respectively. IgGkapp primer corresponded to the constant region of the light chain $\mathrm{CL}$ whereas $\mathrm{IgG} 1 \mathrm{HCl}$ corresponded to the $\mathrm{CH} 1$ gene (sequences are provided in Supplementary file 1: Table S1 at www.actabp.pl). The PCR products were cloned into the pGEM-T easy vector (Promega). Several clones were sequenced and analyzed using vbase2 on-line program (http://www.vbase2. org/vbdnaplot.php) to determine sequences of CDRs of $\mathrm{VH}$ and VL.

Assembling of scFv. Selected functional clones of light and heavy chain of mouse $\operatorname{IgG}$ were used to synthesize $\mathrm{scFv}$ construct. First, to obtain $\mathrm{scFv}$ in $\mathrm{VH}-\mathrm{VL}$ orientation the variable fragments of heavy and light chains were amplified using two pairs of primers either VHNdeF and scFvVHR or scFvVKF and VkNotR. Then VH and VL fragments were assembled into scFv by overlapping PCR reaction using external primers VHNdeF and VkNotR. The same method was applied to synthesize $\mathrm{scF}_{\mathrm{V}}$ in opposite orientation (VL-VH) using specific primers: NdeVLF, linkVLR and linkVHF, VHrevNot. The PCR reactions were performed using Pfu Polymerase (Fermentas). Finally, PCR products were digested with NdeI and NotI restriction enzymes and ligated into the modified pET26b expression vector (Novagen) carrying the sequence encoding His- and Streptags previously cloned by us.

scFv expression in $\boldsymbol{E}$. coli. The scFv constructs were re-cloned from the pET26b into the modified pET201 vector carrying the sequence encoding thioredoxin which resulted in obtaining two recombinant plasmids: pET201/TVHVL and pET201/TVLVH that were used for $\mathrm{scFv}_{\mathrm{v}}$ overexpression in E. coli Origami (DE3) strain. For an efficient production of recombinant TscFvs several different conditions of temperature $\left(37^{\circ} \mathrm{C}, 30^{\circ} \mathrm{C}\right.$, $\left.25^{\circ} \mathrm{C}, 20^{\circ} \mathrm{C}\right)$, IPTG concentration $(10 \mathrm{mM}, 1 \mathrm{mM}$, $0.1 \mathrm{mM}, 0.05 \mathrm{mM}, 0.01 \mathrm{mM}$ ) and time of induction (between $1 \mathrm{~h}-48 \mathrm{~h}$ ) were tested. The TscFv expressed by E. coli were purified on a gravity flow Ni-TED 1000 column (Marcherey-Nagel) in denaturing conditions according to the procedure described in manufacture's manual (Marcherey-Nagel). The first and the second elution fractions were mixed and dialyzed against stepwise decreasing concentration of urea in the range between $6.0-0 \mathrm{M}$. Each step lasted at least 3 hours. Purity of recombinant proteins was evaluated with SDS PAGE.

Phage displayed scFv (PhscFv). The cDNA fragment encoding $\mathrm{scFv}$ in $\mathrm{VL}-\mathrm{VH}$ orientation (without thioredoxin) was amplified by Easy A polymerase (EasyA High-Fidelity PCR Master Mix, Agilent Technologies) with the RRPvuF and VHrevNot primers (sequences are provided in Supplementary file 1: Table S1 at www. actabp.pl) using recombinant plasmid pET26b carrying $\mathrm{scFv}$ cDNA as a template. The obtained PCR product was digested with PvuII and NotI and cloned into pSEX81 phagemid vector (Progen). Small-Scale Phage Rescue was conducted according to the published protocol 154.3.4 by (Dorsam et al., 2002) with minor changes (first centrifugation was prolonged to $25 \mathrm{~min}$ and $5 \mathrm{~h}$ incubation in $37^{\circ} \mathrm{C}$ was changed to $18 \mathrm{~h}$ in $30^{\circ} \mathrm{C}$ ). The obtained phages (PhVLVH) were titrated according to the online protocol 'Helper Phage Production' (Stockinger Lab website: http://www.oardc.ohio-state.edu/stockingerlab/Protocols/HelperPhage.pdf) and used in Phage ELISA. The ELISA was conducted according to (Dor- sam et al., 2002) with minor modifications. Briefly, MaxiSorp plates were coated with $500 \mathrm{ng}$ of the antigen and incubated overnight at $4^{\circ} \mathrm{C}$, washed with PBS, blocked ( 2 h, 2\% skim milk in PBS), incubated with 107-108 pfu for $2 \mathrm{~h}$, washed and incubated with anti-M13 antibodies conjugated with HRP (GE Healthcare).

Sequencing of mAb6-9-1. The amino acid sequence of heavy and light chains of mAb6-9-1 was estimated by mass spectrometry of trypsin-digested $\mathrm{mAb}$ fragments. The analysis was performed in Mass Spectrometry Lab at the Institute of Biochemistry and Biophysics Polish Academy of Sciences, Warsaw.

Hemagglutination inhibition test. HI tests were performed as described previously (Stachyra et al., 2014; Stachyra et al., 2016) using inactivated viruses as antigens, either the highly pathogenic A/Turkey/Poland/35/2007 (H5N1) National Veterinary Research Institute, Pulawy, Poland) or the low pathogenic A/chicken/Belgium/150/1999 (H5N2) (DG Deventer, Netherlands). HI titer was defined as the reciprocal of the highest dilution of sera that completely inhibited hemagglutination.

Antigens and antibodies used in ELISA. The following antigens corresponding to HA from A/swan/ Poland/305-135V08/2006 (H5N1) were used: (1) HA/ Nde, a short variant containing region of 17-340 aa with His-tag at N- terminus produced (in our laboratory) in E. coli; (2) OET, a long variant containing region of $17-$ 530 aa derived from baculovirus system (Oxford Expression Technologies, UK); The following recombinant HA antigens were purchased from Immune Technology Corporation (all of them are 6x His-tagged and derived from 293 cell culture): Qinghai - 17-530 aa from A/Bar-headed Goose/Qinghai/12/05 (H5N1); Vietnam - 17-530 aa from A/VietNam/1203/2004 (H5N1); Anhui - 18-530 aa from A/Anhui/1/2005 (H5N1); Cambodia - 24-341 aa from A/Cambodia/R0405050/2007 (H5N1); Hubei - 18-530 aa from A/Hubei/1/2010 (H5N1); Germany - 17-342 aa from A/Turkey/Germany-MV/R2472/2014 (H5N8); Wuhan - 17-529 aa from A/Wuhan/35995(H3N2); Netherlands - 17-527 aa from A/chicken/ Netherlands/1/03 (H7N7); New Caledonia - 18-530 aa from A/New Caledonia/20/99 (H1N1).

Antibodies. IgY 745 - anty-H5 HA (H5N1) polyclonal IgY was purified in our laboratory from eggs collected from laying hens immunized with DNA vaccine based on pCI vector with cloned cDNA sequence encoding 1-568 aa of HA from A/swan/Poland/305135V08/2006 (H5N1) (Stachyra et al., 2014). IgY was purified using Pierce ${ }^{\mathrm{TM}}$ Chicken IgY purification kit (ThermoFisher). Anti-M13 HRP-conjugated monoclonal antibody (HRP/Anti-M13 Monoclonal Conjugate, GE Healthcare); anti-Strep-tag II monoclonal antibody (StrepMAB- Classic, IBA, GmbH); anti - chicken IgY (Goat anti-Chicken IgY Fc Secondary Antibody, HRP conjugate, Thermo Scientific); anti-mouse HRP antibody (Anti-Mouse IgG ( $\gamma$-chain specific) - Peroxidase antibody produced in goat, Sigma Aldrich) were purchased as indicated.

Indirect and sandwich ELISA. Indirect ELISA. The 96-well MaxiSorp plates (Nunc, Denmark) were coated overnight at $4^{\circ} \mathrm{C}$ with $300 \mathrm{ng}$ of the respective antigens. Following $4 \mathrm{x}$ washing with PBST buffer $(0.1 \mathrm{M}$ PBS with $0.05 \%$ Tween-20) plates were blocked with $2 \%$ BSA in PBS for $1.5 \mathrm{~h}$. Next, plates were incubated with purified antibody (mAb6-9-1 or TscFv) overnight at $4^{\circ} \mathrm{C}$ and after washing incubated $\left(1.5 \mathrm{~h}, 37^{\circ} \mathrm{C}\right)$ with the antistrep-tag antibody, washed and incubated $\left(1 \mathrm{~h}, 37^{\circ} \mathrm{C}\right)$ with anti-mouse HRP conjugated IgG. In the case of mAb6-9-1 the step with anti-strep incubation was omit- 
ted. Following incubation with TMB (3,3',5,5' tetramethylbenzidine, Sigma-Aldrich) absorbance at $450 \mathrm{~nm}$ was measured. Denatured (reduced) antigens were obtained after incubation with $10 \mathrm{mM}$ DTT for $1 \mathrm{~h}$ at $37^{\circ} \mathrm{C}$ according to (Wu et al., 2014).

Sandwich ELISA. The same plates as in Indirect ELISA were coated with $500 \mathrm{ng}$ of the TscFv. Washing and blocking conditions were as above. Next, plates were incubated with antigens for $1.5 \mathrm{~h}$ at room temperature and after washing incubated $(1.5 \mathrm{~h})$ with $\operatorname{IgY} 745$ anti- $\mathrm{H} 5$ polyclonal $\mathrm{IgY}$ at various dilutions in the range 1:4000 to 1:16000, washed again and incubated (45 min, room temperature) with HRP-conjugated goat anti-chicken IgY Fc (Thermo Scientific) 1:12000. Following incubation with TMB absorbance at $450 \mathrm{~nm}$ was measured.

\section{RESULTS AND DISCUSSION}

\section{Prediction of the epitope recognized by mAb6-9-1}

In order to facilitate mapping of the antigenic sites recognized by $\mathrm{mAb}$ 6-9-1 the structural model of the HA variant (A/swan/Poland/305-135V08/2006 (H5N1)) used for antibody generation was created (Fig. 1a) as described in Materials and Methods.

Two commercial phage display peptide libraries were used for selection of peptides that were reactive against mAb6-9-1. In both of them, the library of random peptides (linear dodeca- or loop-constrained heptapeptides in Ph.D. ${ }^{\text {TM}}$-12 Phage Display Peptide Library or

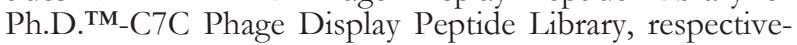
ly) are fused to a minor coat protein pIII of the M13

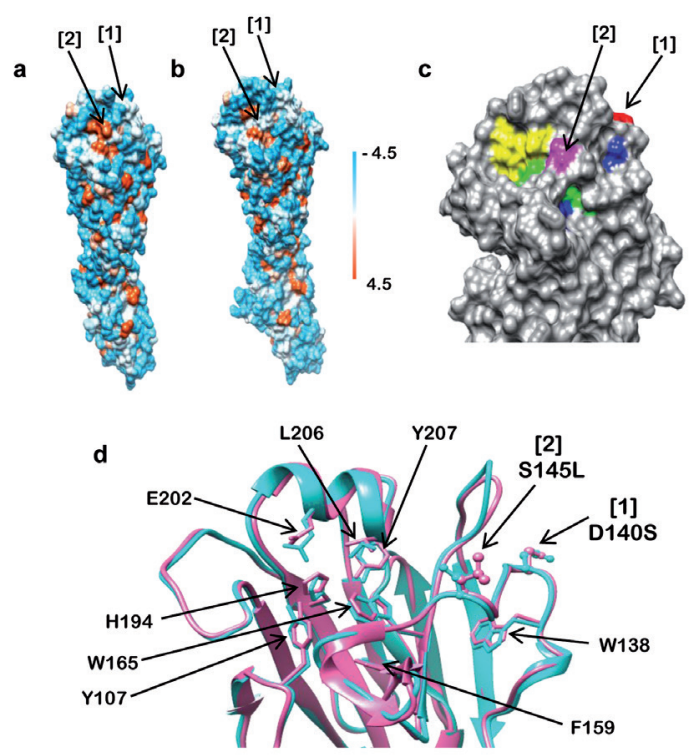

Figure 1. Model of three-dimensional structure of HA from A/swan/Poland/305-135V08/2006 (H5N1) (a) and A/Vietnam/1194/2004 (H5N1) (b).

Both proteins are represented as Connolly surface, colored by amino acids hydrophobicity based on Kyte-Doolittle scale (blue - hydrophilic, red - hydrophobic) (Kyte \& Doolittle, 1982). (c) enlarged head of HA from A/swan/Poland. Yellow surface represents the six amino acids building the RBS interface (Y107, W165, H195, E202, L206, and Y207), dark blue depicts the four residues of the mAb6-9-1 epitope (W138, D140, F159 and W165). W165 is shared between these two groups and is colored green. (d) ribbon diagram of overlapped structures of the part of HA head from Poland (cyan color) and Vietnam (pink color). Two residues defining the binding specificity of mAb6-9-1 are marked on each structure, [1] D140 (Poland)/S (Vietnam), [2] S145 (Poland)/L (Vietnam). phage. After three rounds of panning several potentially positive clones (phage-displayed peptides) were selected for further analysis. First, they were subjected to Phage ELISA to verify their binding capacity with mAb6-9-1, then phage DNA was isolated and sequenced to reveal the predicted amino acid sequence of the mimotopes (polypeptides displayed on the surface of the phages). Phage clones selected from the Ph.D-12 Library were examined in three concentrations: $10^{9}, 10^{8}$ and $10^{7} \mathrm{pfu} /$ well, respectively. The absorbance value (OD450) twofold higher than the value of the negative control was considered positive. The first concentration (109 $\mathrm{pfu} /$ well) was too high, since it resulted in positive signal for all selected clones. The lower concentrations allowed to select 7 clones having the highest reactivity among 21 phages examined (Fig. 2). Six of them expressed the same peptide sequence, VHWDFRQWWQPS, while one different, FPSDWWSQAWSM.

Similar analyses were performed for phage clones selected from the Ph.D-7C Library and resulted in selection of four mimotopes (ETDTLTQ, KTFLSSH, PHKPAMN and KPYTFVG) which occurred with the similar frequency.

The Pepitope server was used for epitope mapping using these affinity-selected peptides and the structural model of HA from the A/Swan/Poland/305135V08/2006 (H5N1). The combined algorithm (consensus results of Mapitope and Pepsurf algorithms) selected one cluster of four HA residues (W138, D140, F159 and W165) from 6 mimotopes that are shown on the structural model (Fig. 1c-d). The Pepitope analysis with only one, (FPSDWWSQAWSM) identified a larger cluster of 8 HA residues (K135, S136, W138, D140, A143, V147, S148 and W165) as an epitope. Both clusters shared three residues (W138, D140 and W165). All of them were localized on the globular head of the HA1 subunit in the proximity of the RBS. Moreover amino acids W138 and D140 were localized within the antigenic epitope called region A, while W165 in the region B. Antigenic epitopes (A-E) were proposed for H5N1

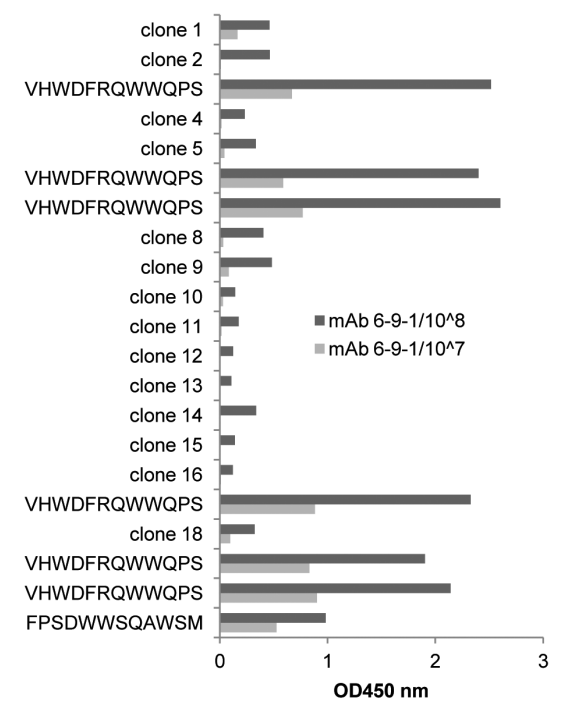

Figure 2. Reactivity of individual phage clones with mAb6-9-1 in Phage ELISA.

Plates were coated with mAb6-9-1 or BSA and to each well $10^{8}$ $\mathrm{pfu}$ and $10^{7} \mathrm{pfu}$ were added, plates were read at $450 \mathrm{~nm}$. For each phage clone signal obtained with target protein (mAb6-91) was compared with that obtained without target protein (e.g. $\mathrm{BSA}$ ); presented on the horizontal axis OD value is reduced by the background (OD value for BSA). For the positive phage clones the amino acid sequences of the dodecapeptides are provided. 
Table 1 The sequences of complementarity determining regions (CDRs) of scFv6-9-1.

\begin{tabular}{llll}
\hline & CDR1 & CDR2 & CDR3 \\
\hline Light chain & SSVNY & YTS & QQFTSSPWT \\
Heavy chain & GYSITSDYA & ISYSGST & ARSGISYYFGTDY \\
\hline
\end{tabular}

Amino acids complementarity which determines CDRs of the VH and VL domains were estimated according to the international ImMunoGeneTics information system ${ }^{\circledast}$ (IMGT, http://www.imgt.org)

highly pathogenic avian influenza viruses based on the classification of the antigenic domains of $\mathrm{H} 3$ (from H3N2 virus) (Peng et al., 2014). Both epitopes, A and B, contrary to the epitopes $\mathrm{C}-\mathrm{E}$, contribute strongly to the antigenic variation of the HPAI (highly pathogenic avian influenza) H5N1 viruses (Peng et al., 2014).

The conclusions from epitope mapping were verified by both, the Hemagglutination Inhibition test and ELI$\mathrm{SA}$ assay. In the HI test ability of mAb6-9-1 to inhibit binding of H5N1 (by RBS) to receptors on erythrocytes surface were analyzed. The HI titer was moderately positive, equal to 32 , for the homologous virus, A/Turkey/ Poland/35/2007(H5N1), while for a nonhomologous virus A/chicken/Belgium/150/1999(H5N2) (DG Deventer, Netherlands) it reached only 8, which was considered as negative. The HI results suggest that the epitope recognized by mAb6-9-1 might be localized nearby the RBS. Similarity between both antigens used in HI test was $86 \%$, besides HA cleavage sites the differences were localized around RBS and the mapped epitope (aa positions: 135, 140, 154, 100, 124, 129, 140, 142, 154, 170, 172, 184, 205, Fig. 3) which may explain the low HI titer obtained for the nonhomologous antigen.

With ELISA assay we analyzed binding of mAb6-9-1 to various $\mathrm{HA}$ antigens from different clades of $\mathrm{H} 5 \mathrm{~N} 1$ viruses as well as $\mathrm{H} 1, \mathrm{H} 3$ and $\mathrm{H} 7 \mathrm{HA}$ from several selected strains (Fig. 4a). The results showed that mAb69-1 can recognize both, a short (HA/Nde) and a long (OET) variants of $\mathrm{H} 5 \mathrm{HA}$ from $\mathrm{A} / \mathrm{swan} /$ Poland/305135V08/2006 (H5N1), clade 2.2 as well as H5 HA from A/Bar-headed Goose/Qinghai/12/05 (H5N1), clade 2.2 and H5 HA from A/Anhui/1/2005 (H5N1), but not the H5 HA from A/Hubei/1/2010 (H5N1), clade 2.3.2.1, A/Cambodia/R0405050/2007 (H5N1), clade 1 nor A/ Turkey/Germany- MV/R2472/2014 (H5N8). The data is in concordance with observation that the most variable inter-clade positions are largely located on the globular head proximal to the RBS (Velkov et al., 2013). We also found that mAb6-9-1 does not recognize the $\mathrm{H} 3$ HA from A/Wuhan/359/95 (H3N2), the H1 HA from A/New Caledonia/20/99 (H1N1) nor the H7 HA from A/chicken/Netherlands/1/03 (H7N7) (Fig. 4a).

Reactivity with $\mathrm{HA} / \mathrm{Nde}$ antigen containing only the HA1 part of H5 HA confirmed that the epitope rec- ognized by mAb6-9-1is located within HA1 subunit (Fig. 4b). Moreover, ELISA confirmed that mAb6-9-1 binds probably a conformational epitope because denaturation of the antigens (reduction of disulfide bonds with $10 \mathrm{mM} \mathrm{DT}^{\circ} \mathrm{T}$ ) resulted in a complete loss of mAb69-1 binding (Fig. 4b).

Comparison of the 3D structures of $\mathrm{H} 5 \mathrm{HA}$ proteins from A/swan/Poland/305-135V08/2006 (H5N1) and from A/Vietnam/1194/2004 (H5N1) (Fig. 1a, b and d) and an alignment of amino acid sequences of the H5 HA antigens tested by ELISA and in HI tests (Fig. 3) clearly showed that two amino acids, D140 and S145 in the proximity of the predicted epitope might decide about antigen binding specificity of mAb6-9-1. None of the analyzed HA proteins with the residue other than aspartic acid in the position [1] i.e. D140 and other than serine in the position [2] i.e. S145 was recognized by mAb6-9-1.

\section{Construction and expression of the scFv - mAb6-9-1 derivative}

The cDNA fragments encoding variable regions of heavy and light chains of mAb6-9-1 were cloned into the pGEM Teasy vector and sequenced. Each of four selected clones of $\mathrm{VH}$ carried the same (functional) sequence. Similarly, one functional sequence was found among thirty analyzed clones carrying VL, however, majority of them were classified as nonfunctional due to an internal stop codon in CDR3. Taking into account the nature of hybridoma cells such finding is not surprising and was reported previously (Toleikis \& Frenzel, 2012). The predicted amino acid sequences of the CDRs in both variable chains are shown in Table 1. Sequences encoding VL and VH were assembled in two possible orientations (VL-VH and VH-VL) with the flexible linker (Gly4Ser) 3 into $\mathrm{scFv}$ and cloned into the modified pET201vector downstream the sequence encoding thioredoxin (Supplementary file Fig. S1 at www.actabp. $\mathrm{pl})$. The choice of thioredoxin as the fusion partner was based on the literature data indicating that the formation of disulfide was essential for many scFvs described (Jurado et al., 2006; Sonoda et al., 2010).

Finally, two recombinant variants of $\mathrm{scFv}$ in fusion with thioredoxin (TscFv: TVHVL and TVLVH) were

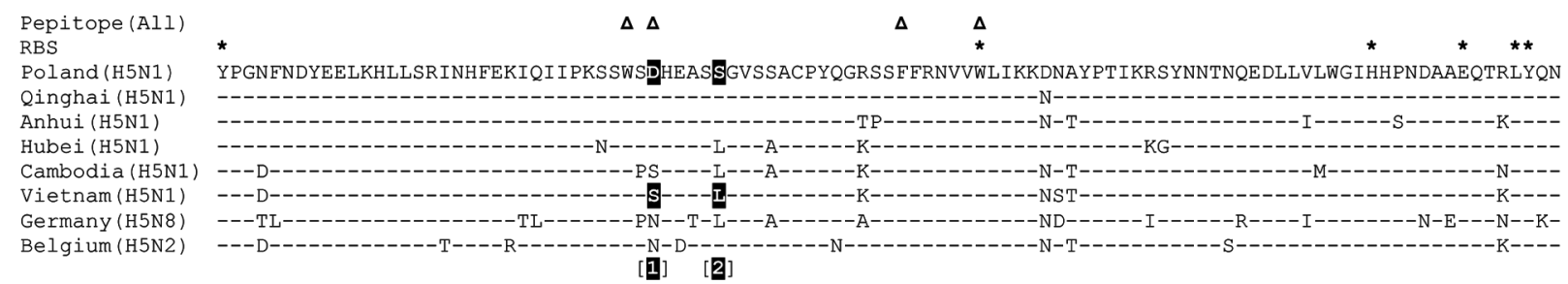

Figure 3. Alignment of the fragments of HA antigens in the region adjacent to the mapped epitope of mAb6-9-1.

The residues corresponding to those forming the RBS according to (Al-Majhdi, 2007) and those identified by the Pepitope software (W138, D140, F159, W165) as the potential target of mAb6-9-1 are marked above the alignment by asterisks and triangles, respectively. The positions that seem to define binding specificity of mAb6-9-1 are marked as white letters on a black background and additionally identified as [1] and [2] below the alignment. Belgium (H5N2) was used only in HI test. For the full names of the viruses refer to the text. 
a

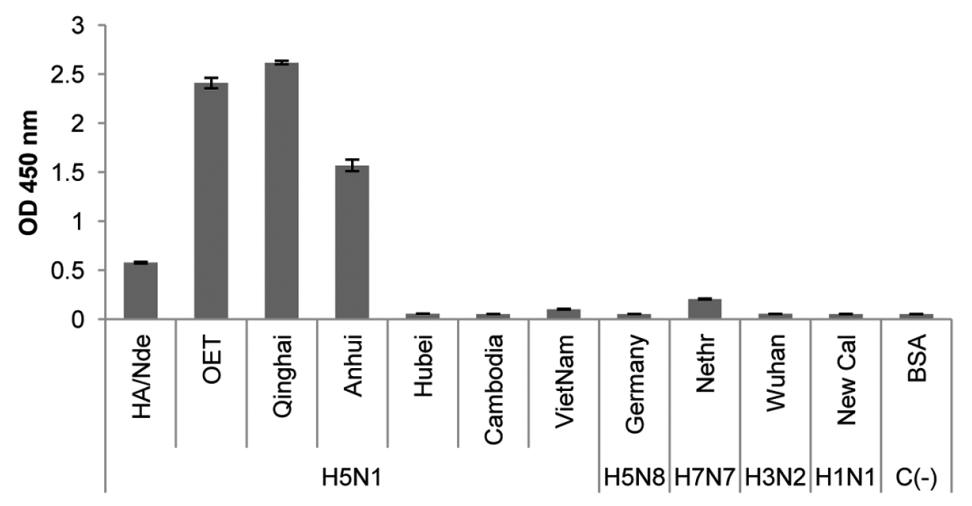

b

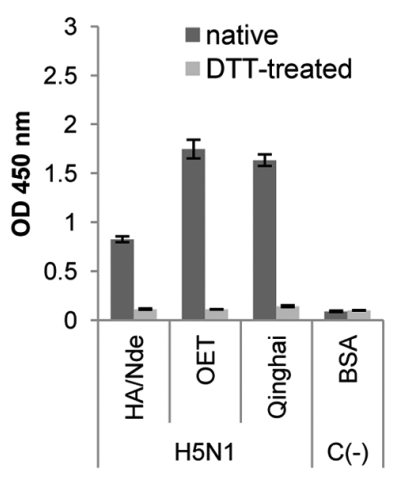

Figure 4. Reactivity of mAb6-9-1.

(a) Indirect ELISA with HA antigens in the native form. (b) Indirect ELISA with the selected HA antigens in the native and the reduced forms. $C(-)$, negative control. The plates were coated with $300 \mathrm{ng}$ of HA from the indicated viruses. Purified mAb6-9-1 and the antimouse IgG conjugated to HRP were used as primary and secondary antibody. The full names of the viruses are provided in the text.
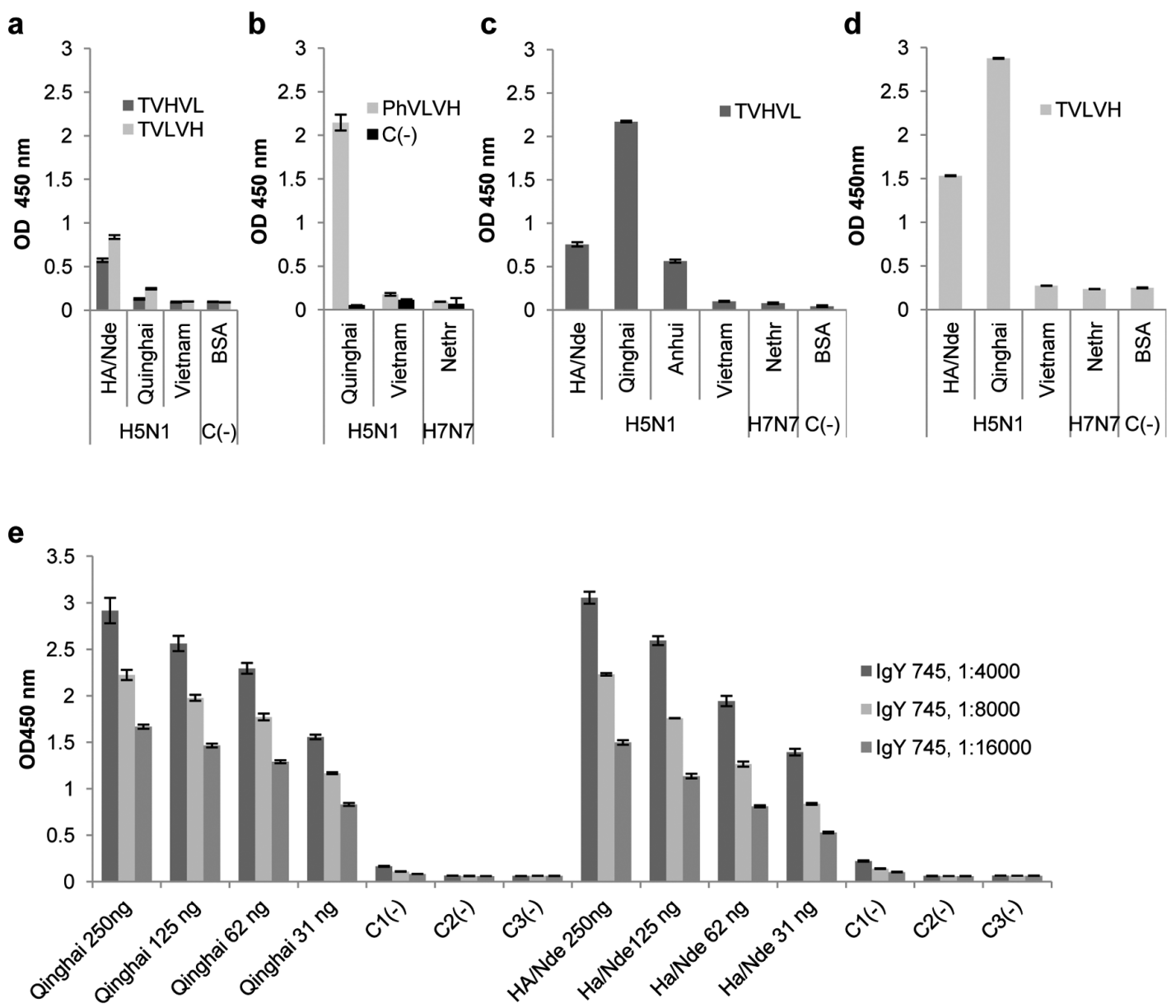

coating: TscFv (TVLVH) 500ng/well

Figure 5. Verification of antigenic specificity of scFv.

(a) Indirect ELISA using TscFv. The plates were coated with $300 \mathrm{ng}$ of the indicated HA antigens or BSA as a negative control, recombinant TscFv (TVHVL, TVLVH) were used as primary antibody, while anti-strep IgG served as secondary antibody. (b) Phage ELISA using phage displayed scFv (PhscFv). Plates were coated with $500 \mathrm{ng}$ of the indicated antigens, incubated with phages (PhVLVH or the negative control) and with anti-M13 lgG as secondary antibody. (c) and (d) sandwich ELISA using TVHVL and TVLVH, respectively. The plates were coated with $500 \mathrm{ng}$ of TVHVL or TVLVH, incubated with the indicated HA antigens (250-31 ng/well) or BSA (c-), negative control, next incubated with anti-H5 HA polyclonal chicken IgY and with anti-chicken IgG-HRP. (e) sandwich ELISA using TVLVH. The plates were coated with $500 \mathrm{ng} /$ well of TVLVH, concentrations of HA antigens and dilutions of anti-H5 HA polyclonal chicken IgY were as indicated. $\mathrm{C} 1(-), \mathrm{C} 2(-)$ and $\mathrm{C} 3(-)$ various negative controls: ELISA performed without HA antigen, without IgY 745, and without antigen and IgY745, respectively. 
produced in E. coli and purified on Ni-TDA agarose. SDS-PAGE analysis indicated the high purity of TscFvs obtained and their expected molecular weights to be about $42 \mathrm{kDa}$ (Supplementary file Fig. S1b at www.actabp.pl).

\section{Antigen binding specificity of the recombinant scFv 6-9-1}

Irrespectively of the $\mathrm{TscFv}$ variant, in the indirect ELISA the strongest binding was observed with HA/Nde - short H5 HA (17-340aa) from A/swan/ Poland/305-135V08/2006 (H5N1) (Fig. 5a). Surprisingly, the $\mathrm{TscF} v$ failed to recognize $\mathrm{H} 5 \mathrm{HA}$ from A/Bar-headed Goose/Qinghai/12/05 (H5N1), the protein previously very well recognized by mAb6-9-1 (Fig. 4a and 5a). Higher plasticity of scFvs in comparison to the fullsize antibodies may affect their specificity and affinity (Shepelyakovskaya et al., 2011). To verify if this change of specificity was due to incorrect $\mathrm{scFv}$ folding a phage displayed variant of $\mathrm{scF}_{\mathrm{V}}(\mathrm{PhVLVH})$ was obtained. Published data showed that the conversion of $\mathrm{scFv}$ from phage-bound to soluble form may results in change of specificity because the phage pIII protein may provide structural support for antigen- binding site (Kaku et al., 2012). The results of phage ELISA (Fig. 5b) showed that indeed PhVLVH was able to specifically recognize the HA antigen from A/Bar-headed Goose/Qinghai/12/05 (H5N1). These results strongly support the conclusion that the cloned cDNA encoding the variable regions of heavy and light chains correspond to the heavy and light chains of mAb6-9-1. Moreover, comparison of the amino acids sequences determined by mass spectroscopy of trypsin-digested mAb6-9-1 with CDRs sequences of $\mathrm{VH}$ and VL predicted from nucleotide sequences of cloned scFv showed $87 \%$ and $100 \%$ identity, respectively (Supplementary file Fig. S2). The alteration of antigen specificity observed by indirect ELISA prompted us to search for a better method of testing the activity of TscFv.

We performed sandwich ELISA using TscFvs (TVHVL and TVLVH, 500ng/well) as capture antibodies, anti-H5 HA IgY 745 as detection antibody and various HA antigens. TscFvs were capable of targeting not only the short fragment of $\mathrm{H} 5 \mathrm{HA}$ (HA/Nde) but also the long fragment of H5 HA from A/Bar-headed Goose/Qinghai/12/05 (H5N1) (Fig. 5c-d).

The H5 HA from A/Anhui/1/2005 (H5N1; clade 2.3.4) was also weakly recognized by $\mathrm{TscFv}$, while binding to H5 HA from A/Vietnam/1203/2004 (H5N1; clade 1) and to $\mathrm{H} 7 \mathrm{HA}$ from $\mathrm{A} /$ chicken/Netherlands/1/03 (H7N7) was not detected (Fig. 5c-d). Obtained results showed that binding capacity of $\mathrm{TscF}$ v to the long $\mathrm{H} 5 \mathrm{HA}$ version may be stronger than to the short H5 HA (HA/Nde) (Fig. 5c-d). This observation was confirmed in detailed analysis when serial two fold dilutions of both HA antigens in the range of 250-30 ng/well and anti-H5 HA IgY (1:4000-1:16000) were applied (Fig. 5e). All data from sandwich ELISA were in accordance with the results obtained for mAb6-9-1 (Fig. 4a).

The observed discrepancies of reactivity of $\mathrm{TscFv}$ toward the long version of $\mathrm{H} 5 \mathrm{HA}$, in two kinds of ELI$\mathrm{SA}$, indirect and sandwich (Fig. 5a and 5c, d, e), may be explained by technical inconsistencies. The lack of binding to the long antigen was observed in the indirect assay, when HA antigens were used to coat the plates and the $\mathrm{TscF}_{\mathrm{V}}$ bound to the HA antigen was detected by the anti-Strep antibody. However, the binding with the same antigen was observed in the sandwich ELISA, when $\mathrm{TscFv}$ was used to coat the plates and HA antigens bound to $\mathrm{TscFv}_{\mathrm{v}}$ were recognized by the specific polyclonal anti-HA IgY antibody, detected subsequently by secondary anti-chicken $\mathrm{IgY}$ antibody conjugated with HRP. Due to the high plasticity of $\mathrm{scFv}_{\mathrm{v}}$, in the indirect ELISA, the Strep-tag was probably hidden in the conformational structure of $\mathrm{TscFv}_{\mathrm{V}}$ and was not accessible for the anti-Strep antibody.

In conclusion, epitope recognized by the full-length monoclonal antibody was mapped in the proximity of the RBS. This assumption is supported by the results of $\mathrm{HI}$ assay. Unfortunately, contrary to mAb6-9-1, TscFv did not demonstrate HI activity (not shown). However, such result does not exclude that $\mathrm{scFv}$ recognized the same epitope as mAb. To prevent hemagglutination, antibody must bind to or near RBS and cause steric hindrance (Ascione et al., 2009; Zhang et al., 2013). It is more likely that the reduced size of $\mathrm{scFv}_{\mathrm{V}}$ is not an effective barrier between virus and the red cell. Both antibodies display narrow specificity to H5 HA from H5N1 influenza viruses, mostly from clade 2.2 and might be used effectively in immunosensors specifically detecting these type of viruses (Jarocka et al., 2014; Jarocka et al., 2016).

\section{Acknowledgements}

The authors thank Dr. Tomasz Sarnowski (IBB PAN) for the thioredoxin vector, Agata Malinowska for LC-MS analysis, and Anna Stachyra, Dr Anna Olszewska, Prof. Zenon Minta and Prof. Krzysztof Śmietanka for sharing the results of $\mathrm{HI}$ test.

\section{Acknowledgements of financial support}

This work was done in frame of the Polish Vaccine Consortium and it was supported by EC Innovative Economy Program, POIG.01.01.02-00-007/08.

\section{REFERENCES}

Al-Majhdi F (2007) Structure of the sialic acid binding site in influenza A virus: hemagglutinin. J Biol Sci 7: 113-122

Ascione A, Capecchi B, Campitelli L, Imperiale V, Flego M, Zamboni S, Gellini M, Alberini I, Pittiglio E, Donatelli I, Temperton NJ, Cianfriglia M (2009) Human monoclonal antibodies in single chain fragment variable format with potent neutralization activity against influenza virus H5N1. Antiviral Res 83: 238-244. doi: 10.1016/j.antiviral.2009.05.005

Bublil EM, Freund NT, Mayrose I, Penn O, Roitburd-Berman A, Rubinstein ND, Pupko T, Gershoni JM (2007) Stepwise prediction of conformational discontinuous B-Cell Epitopes using the mapitope algorithm. Proteins-Structure Function and Bioinformatics 68: 294-304. doi: $10.1002 /$ prot. 21387

Cao Y, Marks JD, Huang Q, Rudnick SI, Xiong C, Hittelman WN, Wen X, Marks JW, Cheung LH, Boland K, Li C, Adams GP, Rosenblum MG (2012a) Single-chain antibody-based immunotoxins targeting Her2/neu: design optimization and impact of affinity on antitumor efficacy and off-target toxicity. Mol Cancer Therap 11: 143153. doi: 10.1158/1535-7163.MCT-11-0519

Cao Z, Meng J, Li X, Wu R, Huang Y, He Y (2012b) The epitope and neutralization mechanism of AVFluIgG01, a broad-reactive human monoclonal antibody against H5N1 influenza virus. Plos One 7. doi: 10.1371/journal.pone.0038126

Choo AB, Dunn RD, Broady KW, Raison RL (2002) Soluble expression of a functional recombinant cytolytic immunotoxin in insect cells. Protein Expr Purif 24: 338-347. doi: 10.1006/prep.2001.1589

Dobhal S, Chaudhary VK, Singh A, Pandey D, Kumar A, Agrawal S (2013) Expression of recombinant antibody (single chain antibody fragment) in transgenic plant Nicotiana tabacum cv. Xanthi. Mol Biol Rep 40: 7027-7037. doi: 10.1007/s11033-013-2822-x

Dorsam H, Braunagel M, Kleist C, Moynet D, Welschof M (2002) Screening of phage displayed antibody libraries. The Protein Protocols Handbook 1073-1082

Du L, Jin L, Zhao G, Sun S, Li J, Yu H, Li Y, Zheng B-J, Liddington RC, Zhou Y, Jiang S (2013) Identification and structural characterization of a broadly neutralizing antibody targeting a novel con- 
served epitope on the influenza virus H5N1 hemagglutinin. I Virol 87: 2215-2225

Ho M, Nagata S, Pastan I (2006) Isolation of anti-CD22 Fv with high affinity by Fv display on human cells. Proc Natl Acad Sci U S A 103: 9637-9642

Jarocka U, Sawicka R, Gora-Sochacka A, Sirko A, Dehaen W, Radecki J, Radecka H (2016) An electrochemical immunosensor based on a4,4-thiobisbenzenethiol self-assembled monolayer for the detectionof hemagglutinin from avian influenza virus H5N1. Sensors and Acttuators B: Chemical 228: 25-30

Jarocka U, Sawicka R, Góra-Sochacka A, Sirko A, Zagórski-Ostoja W, Radecki J, Radecka H (2014) An immunosensor based on antibody binding fragments attached to gold nanoparticles for the detection of peptides derived from avian influenza hemagglutinin H5. Sensors (Basel) 14: 15714-15728. doi: 10.3390/s140915714

Jurado P, de Lorenzo V, Fernandez LA (2006) Thioredoxin fusions increase folding of single chain $F_{V}$ antibodies in the cytoplasm of Escherichia coli: Evidence that chaperone activity is the prime effect of thioredoxin. J Mol Biol 357: 49-61. doi: 10.1016/j.jmb.2005.12.058

Kaku Y, Noguchi A, Okutani A, Inoue S, Tanabayashi K, Yamamoto Y, Hotta A, Suzuki M, Sugiura N, Yamada A (2012) Altered specificity of single-chain antibody fragments bound to pandemic H1N12009 influenza virus after conversion of the phage-bound to the soluble form. BMC Res Notes 5: 483. doi: 10.1186/1756-0500-5-483

Kalenik B, Sawicka R, Góra-Sochacka A, Sirko A (2014) Influenza prevention and treatment by passive immunization. Acta Biochim Pol 61: $573-587$

Kyte J, Doolittle RF (1982) A simple method for displaying the hydropathic character of a protein. J Mol Biol 157: 105-132. 10.1016/00222836(82)90515-0

Ladiges W, Osman GE (2001)Molecular characterization of immunoglobulin genes. In Basic Methods in Antibody Production and Characterization. Howard GC \& Bethell D eds, pp 169-192: CRC Press LLC

Li Y, Zhang X, Xu Q, Fu Q, Zhu Y, Chen S, Peng D, Liu X (2013) Characterisation and haemagglutinin gene epitope mapping of a variant strain of $\mathrm{H} 5 \mathrm{~N} 1$ subtype avian influenza virus. Vet Microbiol 162: 614-622

Mayrose I, Penn O, Erez E, Rubinstein ND, Shlomi T, Freund NT, Bublil EM, Ruppin E, Sharan R, Gershoni JM, Martz E, Pupko T (2007a) Pepitope: epitope mapping from affinity-selected peptides. Bioinformatics 23: 3244-3246. doi: 10.1093/bioinformatics/btm493

Mayrose I, Shlomi T, Rubinstein ND, Gershoni JM, Ruppin E, Sharan R, Pupko T (2007b) Epitope mapping using combinatorial phagedisplay libraries: a graph-based algorithm. Nucleic Acids Research 35: 69-78. doi: 10.1093/nar/gk1975

Meng EC, Pettersen EF, Couch GS, Huang CC, Ferrin TE (2006) Tools for integrated sequence-structure analysis with UCSF Chimera. Bmc Bioinformatics 7. doi: 10.1186/1471-2105-7-339

Oriuchi N, Higuchi T, Hanaoka H, Iida Y, Endo K (2005) Current status of cancer therapy with radiolabeled monoclonal antibody. Ann Nucl Med 19: 355-365

Peng Y, Zou Y, Li H, Li K, Jiang T (2014) Inferring the antigenic epitopes for highly pathogenic avian influenza H5N1 viruses. Vaccine 32: 671-676. doi: 10.1016/j.vaccine.2013.12.005

Rouet R, Lowe D, Dudgeon K, Roome B, Schofield P, Langley D, Andrews J, Whitfeld P, Jermutus L, Christ D (2012) Expression of high-affinity human antibody fragments in bacteria. Nature Protocols 7: 364-373. doi: 10.1038/nprot.2011.448
Sali A, Blundell TL (1993) Comparative protein modeling by satisfaction of spatial restraints. J Mol Biol 234: 779-815. doi: 10.1006/ jmbi.1993.1626

Shepelyakovskaya AO, Laman AG, Lomonosova AV, Fursova KK, Savinov GV, Vertiev YV, Brovko FA, Grishin EV (2011) Effect of the format of antibodies on their specificity. Mol Immunol 49: 433440. doi: 10.1016/j.molimm.2011.09.017

Smith GJD, Donis RO, Working WOFHNE (2012) Continued evolution of highly pathogenic avian influenza A (H5N1): updated nomenclature. Influenza and Other Respiratory Viruses 6: 1-5. doi: 10.1111/j.1750-2659.2011.00298.x

Smith GJD, Donis RO, World Health Organization W (2015) Nomenclature updates resulting from the evolution of avian influenza $\mathrm{A}(\mathrm{H} 5)$ virus clades 2.1.3.2a, 2.2.1, and 2.3.4 during 2013-2014. Influenza and Other Respiratory Viruses 9: 271-276. doi: 10.1111/irv.12324

Sonoda H, Kumada Y, Katsuda T, Yamaji H (2010) Functional expression of single-chain $F_{V}$ antibody in the cytoplasm of Escherichia coli by thioredoxin fusion and co-expression of molecular chaperones. Protein Expression and Purification 70: 248-253. doi: 10.1016/j. pep.2009.11.003

Stachyra A, Gora-Sochacka A, Sawicka R, Florys K, Saczynska V, Olszewska M, Pikula A, Smietanka K, Minta Z, Szewczyk B, Zagorski W, Sirko A (2014) Highly immunogenic prime-boost DNA vaccination protects chickens against challenge with homologous and heterologous H5N1 virus. Trials V accinol 3: 40-46

Stachyra A, Redkiewicz P, Kosson P, Protasiuk A, Góra-Sochacka A, Kudla G, Sirko A (2016) Codon optimization of antigen coding sequences improves the immune potential of DNA vaccines against avian influenza virus H5N1 in mice and chickens. Virol J 13: 143. doi: 10.1186/s12985-016-0599-y

Szewczyk B, Bienkowska-Szewczyk K, Krol E (2014) Introduction to molecular biology of influenza A viruses. Acta Biochim Pol 61: $397-$ 401

Tan Y, Ng Q, Jia Q, Kwang J, He F (2015) A novel humanized antibody neutralizes $\mathrm{H} 5 \mathrm{~N} 1$ influenza virus via two different mechanisms. J Virol 89: 3712-3722. doi:10.1128/JVI.03014-14

Toleikis L, Frenzel A (2012) Cloning single-chain antibody fragments $(\mathrm{ScFv})$ from hyrbidoma cells. Methods Mol Biol 907: 59-71

Velkov T, Ong C, Baker MA, Kim H, Li J, Nation RL, Huang JX, Cooper MA, Rockman S (2013) The antigenic architecture of the hemagglutinin of influenza H5N1 viruses. Mol Immunol 56: 705-719. doi: 10.1016/j.molimm.2013.07.010

Wu R, Li X, Leung H-C, Cao Z, Qiu Z, Zhou Y, Zheng B-J, He $Y$ (2014) A novel neutralizing antibody against diverse clades of H5N1 influenza virus and its mutants capable of airborne transmission. Antiviral Res 106: 13-23. http://dx.doi.org/10.1016/j.antiviral.2014.03.005

Zhang X, Qi X, Zhang Q, Zeng X, Shi Z, Jin Q, Zhan F, Xu Y, Liu Z, Feng Z, Jiao Y (2013) Human 4F5 single-chain Fv antibody recognizing a conserved HA1 epitope has broad neutralizing potency against H5N1 influenza A viruses of different clades. Antiviral Res 99: 91-99. http://dx.doi.org/10.1016/j.antiviral.2013.05.001

Zuo T, Sun J, Wang G, Jiang L, Zuo Y, Li D, Shi X, Liu X, Fan S, Ren H, Hu H, Sun L, Zhou B, Liang M, Zhou P, Wang X, Zhang $\mathrm{L}$ (2015) Comprehensive analysis of antibody recognition in convalescent humans from highly pathogenic avian influenza H5N1 infection. Nat Commun 6: 8855-8867. doi: 10.1038/ncomms9855 